\title{
Identities of symmetry for Bernoulli polynomials and power sums
}

\author{
Taekyun Kim', Dae San Kim², Han Young Kim and Jongkyum Kwon ${ }^{3 *}$
}

\section{"Correspondence:}

mathkjk26@gnu.ac.kr

${ }^{3}$ Department of Mathematics

Education and ERI, Gyeongsang

National University, Jinju 52828,

Republic of Korea

Full list of author information is

available at the end of the article

\begin{abstract}
Identities of symmetry in two variables for Bernoulli polynomials and power sums had been investigated by considering suitable symmetric identities. T. Kim used a completely different tool, namely the $p$-adic Volkenborn integrals, to find the same identities of symmetry in two variables. Not much later, it was observed that this $p$-adic approach can be generalized to the case of three variables and shown that it gives some new identities of symmetry even in the case of two variables upon specializing one of the three variables. In this paper, we generalize the results in three variables to those in an arbitrary number of variables in a suitable setting and illustrate our results with some examples.
\end{abstract}

MSC: $11 \mathrm{~B} 68 ; 11 \mathrm{~S} 80 ; 05 \mathrm{~A} 19$

Keywords: Bernoulli polynomial; Power sum; p-adic Volkenborn integral

\section{Introduction and preliminaries}

Tuenter [17] obtained the following identity of symmetry involving the Bernoulli numbers and the power sums. This was done by showing that the exponential generating function of the sum on the left-hand side of (1) is invariant under the interchange of $w_{1}$ and $w_{2}$.

$$
\sum_{i=0}^{n}\left(\begin{array}{c}
n \\
i
\end{array}\right) B_{i} S_{n-i}\left(w_{1}-1\right) w_{1}^{i-1} w_{2}^{n-i}=\sum_{i=0}^{n}\left(\begin{array}{c}
n \\
i
\end{array}\right) B_{i} S_{n-i}\left(w_{2}-1\right) w_{2}^{i-1} w_{1}^{n-i}
$$

where $w_{1}, w_{2}$ are any positive integers, $n$ is a nonnegative integer, $B_{n}$ are Bernoulli numbers in (8), $S_{k}(n)$ are the power sums in (10).

When $w_{2}=1$, equation (1) reduces to the following recurrence relation for the Bernoulli numbers:

$$
B_{n}=\frac{1}{w_{1}\left(1-w_{1}^{n}\right)} \sum_{i=0}^{n-1}\left(\begin{array}{l}
n \\
i
\end{array}\right) B_{i} S_{n-i}\left(w_{1}-1\right) w_{1}^{i},
$$

which was proved by Deeba and Rodriguez [2] and Gessel [3]. Actually, it was a conjecture posed by Namias who found identity (2) for $w_{1}=2,3$ by using the multiplication formula for the gamma function (see [2]).

(c) The Author(s) 2020. This article is licensed under a Creative Commons Attribution 4.0 International License, which permits use, sharing, adaptation, distribution and reproduction in any medium or format, as long as you give appropriate credit to the original author(s) and the source, provide a link to the Creative Commons licence, and indicate if changes were made. The images or other third party material in this article are included in the article's Creative Commons licence, unless indicated otherwise in a credit line to the material. If material is not included in the article's Creative Commons licence and your intended use is not permitted by statutory regulation or exceeds the permitted use, you will need to obtain permission directly from the copyright holder. To view a copy of this licence, visit http://creativecommons.org/licenses/by/4.0/. 
A completely different approach to identities of symmetry was given in [12], where the $p$-adic Volkenborn integrals for uniformly differential functions (see (5)) were used. In particular, the following two identities were obtained:

$$
\begin{aligned}
& \sum_{i=0}^{n}\left(\begin{array}{l}
n \\
i
\end{array}\right) B_{i}\left(w_{2} x\right) S_{n-i}\left(w_{1}-1\right) w_{1}^{i-1} w_{2}^{n-i}=\sum_{i=0}^{n}\left(\begin{array}{c}
n \\
i
\end{array}\right) B_{i}\left(w_{1} x\right) S_{n-i}\left(w_{2}-1\right) w_{2}^{i-1} w_{1}^{n-i}, \\
& \sum_{i=0}^{w_{1}-1} B_{n}\left(w_{2} x+\frac{w_{2}}{w_{1}} i\right) w_{1}^{n-1}=\sum_{i=0}^{w_{2}-1} B_{n}\left(w_{1} x+\frac{w_{1}}{w_{2}} i\right) w_{2}^{n-1} .
\end{aligned}
$$

We note here that (3) becomes (1) when $x=0$, and that (3) and (4) can be generalized to higher-order Bernoulli polynomials (see [18]). It turns out that this $p$-adic approach to identities of symmetry has the merit of being easily generalized. In addition, the identities of symmetry can be found also for Euler polynomials [9], $q$-Bernoulli polynomials [5, 13, 14 ], and $q$-Euler polynomials [6], respectively by using $p$-adic fermionic integrals, $p$-adic $q$-Volkenborn integrals, and $p$-adic fermionic $q$-integrals [11]. For the $q$-Bernoulli and $q$ Euler polynomials, we let the reader refer to the papers $[1,4,16]$. Indeed, in [10] many identities of symmetry for three variables were obtained for the first time by adopting the $p$-adic Volkenborn integral approach initiated in [12] (see Example 3.1). As was mentioned in Sect. 1 of [10], by specializing the variable $w_{3}$ as 1 in (c-1), (c-2), and (c-3) of Example 3.1, it was shown that (3) and (4) are all equal and that they are further equal to the following identities:

$$
\begin{aligned}
& \sum_{k+\ell+m=n}\left(\begin{array}{c}
n \\
k, \ell, m
\end{array}\right) B_{k}\left(y_{1}\right) S_{\ell}\left(w_{1}-1\right) S_{m}\left(w_{2}-1\right) w_{1}^{k+m-1} w_{2}^{k+\ell-1} \\
& =w_{1}^{n-1} \sum_{k=0}^{n}\left(\begin{array}{l}
n \\
k
\end{array}\right) \sum_{i=0}^{w_{1}-1} B_{k}\left(y_{1}+\frac{i}{w_{1}}\right) S_{n-k}\left(w_{2}-1\right) w_{2}^{k-1} \\
& =w_{2}^{n-1} \sum_{k=0}^{n}\left(\begin{array}{l}
n \\
k
\end{array}\right) \sum_{i=0}^{w_{2}-1} B_{k}\left(y_{1}+\frac{i}{w_{2}}\right) S_{n-k}\left(w_{1}-1\right) w_{1}^{k-1} \\
& =\left(w_{1} w_{2}\right)^{n-1} \sum_{i=0}^{w_{1}-1} \sum_{j=0}^{w_{2}-1} B_{n}\left(y_{1}+\frac{i}{w_{1}}+\frac{j}{w_{2}}\right) .
\end{aligned}
$$

Thus we may say that the abundance of symmetries in (c-1), (c-2), and (c-3) shed new light even on the existing identities in two variables. These would not be unearthed if more symmetries had not been available. Moreover, the identities of symmetry for higherorder Bernoulli polynomials in two variables in [18], which was done by manipulations of identities, can be done by using the $p$-adic Volkenborn integrals so as to give abundant symmetries in three variables (see $[7,8]$ ).

The aim of this paper is to generalize the results in three variables [10] to those in an arbitrary number of variables in a suitable setting. Theorem 2.2 is the main result of this paper. Further, we illustrate our results with examples in Sect. 3. In the rest of this section, we recall the facts that are needed throughout this paper.

Let $p$ be a fixed prime. Throughout this paper, $\mathbb{Z}_{p}, \mathbb{Q}_{p}, \mathbb{C}_{p}$ will respectively denote the ring of $p$-adic integers, the field of $p$-adic rational numbers, and the completion of the 
algebraic closure of $\mathbb{Q}_{p}$. For a uniformly differentiable function $f: \mathbb{Z}_{p} \rightarrow \mathbb{C}_{p}$, the $p$-adic Volkenborn integral of $f$ is defined by

$$
\int_{\mathbb{Z}_{p}} f(z) d \mu(z)=\lim _{N \rightarrow \infty} \frac{1}{p^{N}} \sum_{j=0}^{p^{N}-1} f(j) .
$$

Then it is easy to see that

$$
\int_{\mathbb{Z}_{p}} f(z+1) d \mu(z)=\int_{\mathbb{Z}_{p}} f(z) d \mu(z)+f^{\prime}(z) .
$$

Let $|\cdot|_{p}$ be the normalized non-Archimedean absolute value of $\mathbb{C}_{p}$ such that $|p|_{p}=\frac{1}{p}$, and let

$$
E=\left\{\left.t \in \mathbb{C}_{p}|| t\right|_{p}<p^{-\frac{1}{p-1}}\right\}
$$

Then, for each fixed $t \in E$, the function $f(z)=e^{z t}$ is analytic on $\mathbb{Z}_{p}$, and by applying (6) to this $f$, we get the $p$-adic integral expression of the generating function for Bernoulli numbers $B_{n}$ given by

$$
\int_{\mathbb{Z}_{p}} e^{z t} d \mu(z)=\frac{t}{e^{t}-1}=\sum_{n=0}^{\infty} B_{n} \frac{t^{n}}{n !} \quad(t \in E) .
$$

So we have the following $p$-adic integral expression of the generating function for the Bernoulli polynomials $B_{n}(x)$ :

$$
\int_{\mathbb{Z}_{p}} e^{(x+z) t} d \mu(z)=\frac{t}{e^{t}-1} e^{x t}=\sum_{n=0}^{\infty} B_{n}(x) \frac{t^{n}}{n !} \quad\left(t \in E, x \in \mathbb{Z}_{p}\right) .
$$

Here and throughout this paper, we will have many instances to be able to interchange integral and infinite sum. That is justified by Proposition 55.4 in [15]. Let $S_{k}(n)$ denote the $k$ th power sum of the first $n+1$ nonnegative integers, namely

$$
S_{k}(n)=\sum_{i=0}^{n} i^{k}=0^{k}+1^{k}+\cdots+n^{k}
$$

In particular, we have

$$
S_{0}(n)=n+1, \quad S_{k}(0)= \begin{cases}1 & \text { for } k=0, \\ 0 & \text { for } k>0 .\end{cases}
$$

From (8) and (10), one easily derives the following identities: for $w \in \mathbb{Z}_{>0}$,

$$
\begin{aligned}
\frac{w \int_{\mathbb{Z}_{p}} e^{x t} d \mu(x)}{\int_{\mathbb{Z}_{p}} e^{w y t} d \mu(y)} & =\sum_{i=0}^{w-1} e^{i t} \\
& =\sum_{k=0}^{\infty} S_{k}(w-1) \frac{t^{k}}{k !} \quad(t \in E) .
\end{aligned}
$$


Let $n \geq 2$, and let $I_{n}=\{1,2, \ldots, n\}$. Then the symmetric group $S_{n}$ acts on $I_{n}$ in a natural way as $(\sigma, j) \mapsto \sigma(j)$. For each integer $j$ with $1 \leq j \leq n$, let $\Omega_{j}$ be the subset of $2^{I_{n}}$ consisting of all $j$-element subsets of $I_{n}$.

Proposition 1.1 Let $\Omega$ be a nonempty subset of $2^{I_{n}}$ not containing the empty set $\phi$. Then there is an action of $S_{n}$ on $\Omega$ induced by the natural action of $S_{n}$ on $I_{n}$ if and only if $\Omega=$ $\bigcup_{j \in J} \Omega_{j}$ for some nonempty subset $J$ of $I_{n}$. Moreover, such an action of $S_{n}$ on $\Omega$ is transitive if and only if $\Omega=\Omega_{j}$ for some $j(1 \leq j \leq n)$.

Proof $(\Leftarrow)$ For each $j(1 \leq j \leq n)$, the natural action of $S_{n}$ on $I_{n}$ induces an action of $S_{n}$ on $\Omega_{j}$, which is obviously transitive. In turn, this induces an action of $S_{n}$ on $\Omega=\bigcup_{j \in J} \Omega_{j}$ for some nonempty subset $J$ of $I_{n}$.

$(\Rightarrow)$ Assume that there is an action of $S_{n}$ on $\Omega$ induced by the natural action of $S_{n}$ on $I_{n}$. If $A \in \Omega$, with $|A|=j(1 \leq j \leq n)$, then $\sigma A \in \Omega$ for all $\sigma \in S_{n}$, and hence $\Omega_{j} \subset \Omega$. This shows that $\Omega=\bigcup_{j \in J} \Omega_{j}$ for some nonempty subset $J$ of $I_{n}$. For the second statement, note that the action cannot be transitive if $\Omega=\bigcup_{j \in J} \Omega_{j}$ with $|J| \geq 2$.

\section{Main results}

Assume that $n$ is any fixed integer $\geq 2$. Then we will introduce notations that will be used throughout this paper.

- $I=I_{n}=\{1,2, \ldots, n\}$.

- $\Omega_{j}=\Omega_{j}^{(n)}=$ the set consisting of all $j$-element subsets of $I$ for any $j=1,2, \ldots, n-1$ $\left(\left|\Omega_{j}\right|=\left(\begin{array}{l}n \\ j\end{array}\right)\right)$.

We give the reversed lexicographic ordering on each $\Omega_{j}$ and also on any nonempty subset $\Omega$ of $\Omega_{j}$. For example, $\Omega_{3}=\{\overline{1}=\{2,3,4\}<\overline{2}=\{1,3,4\}<\overline{3}=\{1,2,4\}<\overline{4}=\{1,2,3\}$, when $n=4, j=3 ; \Omega=\{\{2,3,4\}<\{1,2,4\}\}$ is a subset of $\Omega_{j}$. We write $\Omega<\Omega^{\prime}$ for disjoint nonempty subsets $\Omega, \Omega^{\prime}$ of $\Omega_{j}$ if every member of $\Omega$ is smaller than that of $\Omega^{\prime}$. Also, we agree that $\phi<\Omega, \Omega<\phi$ for every (including the empty set) subset $\Omega$ of $\Omega_{j}$.

Every permutation $\sigma$ in $S_{n}$ gives rise to a natural bijection of $\Omega_{j}$ onto itself given by $A \mapsto \sigma A$, where $\sigma A$ is the set obtained from $A$ by applying $\sigma$ to each member of $A$.

- $w_{1}, w_{2}, \ldots, w_{n}$ typical $n$ positive integers.

- $w_{A}=\prod_{j \in A} w_{j}$ for any subset $A$ of $I\left(w_{\phi}=1\right)$. For example, $w_{\{1,2,3\}}=w_{1} w_{2} w_{3}$. Also, we note that $\prod_{A \in \Omega_{j}} w_{A}=w_{I}^{\left(\begin{array}{c}n-1 \\ j-1\end{array}\right)}$.

- $\hat{w}_{i}=\frac{w_{I}}{w_{i}}$.

- $\bar{A}=$ the complement of $A$ in $I$ for any subset $A$ of $I$, so that $w_{A} w_{\bar{A}}=w_{I}$ for any subset $A$ of $I$.

- $\hat{w}_{A}=\prod_{i \in A} \hat{w}_{i}=\left(w_{I}\right)^{|A|-1} w_{\bar{A}}\left(\hat{w}_{I}=\left(w_{I}\right)^{|A|-1}\right)$.

- $x_{A}=x_{j_{1} j_{2} \cdots j_{r}}$ for any nonempty subset $A=\left\{j_{1}, j_{2}, \ldots, j_{r}\right\}$ of $I$ with $j_{1}<j_{2}<\cdots<j_{r}$. For example, $x_{\{1,2,3\}}=x_{123}$. These are typical variables of integration.

- $\phi_{j}: \Omega_{j} \rightarrow \Omega_{n-j}(A \mapsto \bar{A})$ is a bijection.

- $p_{j}(w ; x)=p_{j}^{(n)}(w ; x)=\sum_{A \in \Omega_{j}} w_{A} x_{\bar{A}}$ for $j=1,2, \ldots, n-1$. For example, with $n=4$, we have

$$
\begin{aligned}
& p_{1}(w ; x)=w_{1} x_{234}+w_{2} x_{134}+w_{3} x_{124}+w_{4} x_{123} \\
& p_{2}(w ; x)=w_{1} w_{2} x_{34}+w_{1} w_{3} x_{24}+w_{1} w_{4} x_{23}+w_{2} w_{3} x_{14}+w_{2} w_{4} x_{13}+w_{3} w_{4} x_{12}
\end{aligned}
$$




$$
p_{3}(w ; x)=w_{1} w_{2} w_{3} x_{4}+w_{1} w_{2} w_{4} x_{3}+w_{1} w_{3} w_{4} x_{2}+w_{2} w_{3} w_{4} x_{1}
$$

- $d \mu(\Omega)=\prod_{A \in \Omega} d \mu\left(x_{\bar{A}}\right)$ for any nonempty subset $\Omega$ of $\Omega_{j}$.

As before, assume that $n$ is any fixed integer $\geq 2$ and that $j$ is an integer with $1 \leq j \leq$ $n-1$. Then, in view of Proposition 1.1, for any subset $\Omega$ for $\Omega_{j}$, we consider the following quotients of integrals given by

$$
\begin{aligned}
I_{j}(\Omega) & =I_{j}^{(n)}(\Omega)=\frac{\int_{\mathbb{Z}_{p} \Omega_{j} \mid} e^{\left(p_{j}\left(w_{j} x\right)+w_{I}\left(\sum_{A \in \Omega_{j}-\Omega} y_{\bar{A}}\right)\right) t} d \mu\left(\Omega_{j}\right)}{\left(\int_{\mathbb{Z}_{p}} e^{w_{I} z t} d \mu(z)\right)^{|\Omega|}} \\
& =\frac{w_{I}^{\left({ }_{j-1}^{n-1}\right)-|\Omega|} t^{\left|\Omega_{j}\right|-|\Omega|} e^{w_{I}\left(\sum_{A \in \Omega_{j}-\Omega} y_{\bar{A}}\right) t}\left(e^{w_{I} t}-1\right)^{|\Omega|}}{\prod_{A \in \Omega_{j}}\left(e^{w_{A} t}-1\right)} .
\end{aligned}
$$

Here, we have to observe that

$$
\begin{aligned}
\int_{\mathbb{Z}_{p}^{\left|\Omega_{j}\right|}} e^{p_{j}(w ; x) t} d \mu\left(\Omega_{j}\right) & =\prod_{A \in \Omega_{j}} \int_{\mathbb{Z}_{p}} e^{w_{A} x_{\bar{A}} t} d \mu\left(x_{\bar{A}}\right) \\
& =\prod_{A \in \Omega_{j}} \frac{w_{A} t}{e^{w_{A} t}-1}=\frac{w_{I}^{\left({ }_{j-1}^{n-1}\right)} t^{\left|\Omega_{j}\right|}}{\prod_{A \in \Omega_{j}}\left(e^{w_{A} t}-1\right)} .
\end{aligned}
$$

It is important to observe here, either from (14) or from (15), that the integrals $I_{j}(\Omega)$ are invariant under any permutation of $w_{1}, w_{2}, \ldots, w_{n}$.

Now, we decompose $\Omega$ into a disjoint union $\Omega=\Omega^{(e)} \cup \Omega^{(s)}$ with $\Omega^{(e)}<\Omega^{(s)}$. As we allow either $\Omega^{(e)}$ or $\Omega^{(s)}$ to be the empty set, there are $|\Omega|+1$ ways of doing this. Then, by invoking (12) and (13), we write the integral in (14) as follows:

$$
\begin{aligned}
I_{j}(\Omega)= & \frac{1}{\prod_{A \in \Omega} w_{\bar{A}}} \times \prod_{A \in \Omega_{j}-\Omega} \int_{\mathbb{Z}_{p}} e^{w_{A}\left(x_{\bar{A}}+w_{\bar{A}} y_{\bar{A}}\right) t} d \mu\left(x_{\bar{A}}\right) \times \prod_{A \in \Omega} \frac{w_{\bar{A}} \int_{\mathbb{Z}_{p}} e^{w_{A} x_{\bar{A}} t} d \mu\left(x_{\bar{A}}\right)}{\int_{\mathbb{Z}_{p}} e^{w_{\bar{A}} w_{A} z t} d \mu(z)} \\
= & \frac{1}{\prod_{A \in \Omega} w_{\bar{A}}} \times \prod_{A \in \Omega_{j}-\Omega} \int_{\mathbb{Z}_{p}} e^{w_{A}\left(x_{\bar{A}}+w_{\bar{A}} y_{\bar{A}}\right) t} d \mu\left(x_{\bar{A}}\right) \\
& \times \prod_{A \in \Omega^{(e)}}\left(\sum_{i_{A}=0}^{w_{\bar{A}}-1} e^{i_{A} w_{A} t}\right) \times \prod_{A \in \Omega^{(s)}}\left(\sum_{j_{A}=0}^{\infty} S_{j_{A}}\left(w_{\bar{A}}-1\right) \frac{\left(w_{A} t\right)^{j_{A}}}{j_{A} !}\right) .
\end{aligned}
$$

Note here that we used the identity in (12) for all $A \in \Omega^{(e)}$, and that in (13) for all $A \in \Omega^{(s)}$.

Remark 2.1 We observe that (15) depends essentially only on the size $|\Omega|$ of $\Omega$. Namely, if $\Omega^{(1)}$ and $\Omega^{(2)}$ are two subsets of $\Omega_{j}$, with $\left|\Omega^{(1)}\right|=\left|\Omega^{(2)}\right|$, and $f: \Omega_{j} \rightarrow \Omega_{j}$ is any bijective map sending $\Omega^{(1)}$ onto $\Omega^{(2)}$, then we have

$$
\begin{aligned}
& \frac{w_{I}^{\left(\begin{array}{c}
n-1 \\
j-1
\end{array}\right)-\left|\Omega^{(2)}\right|} t^{\left|\Omega \Omega_{j}\right|-\left|\Omega^{(2)}\right|} e^{w_{I}\left(\sum_{A \in \Omega_{j}-\Omega^{(2)}} y_{\bar{A}}\right) t}\left(e^{w_{I} t}-1\right)^{\left|\Omega^{(2)}\right|}}{\prod_{A \in \Omega_{j}}\left(e^{w_{A} t}-1\right)} \\
& =\frac{w_{I}^{\left(\begin{array}{l}
(j-1) \\
j-1
\end{array}\right)-\left|\Omega^{(1)}\right|} t^{\left|\Omega \Omega_{j}\right|-\left|\Omega^{(1)}\right|} e^{w_{I}\left(\sum_{A \in \Omega_{j}-\Omega^{(1)}} y_{f(A)}\right) t}\left(e^{w_{I} t}-1\right)^{\left|\Omega^{(1)}\right|}}{\prod_{A \in \Omega_{j}}\left(e^{w_{A} t}-1\right)} .
\end{aligned}
$$


Thus (14) with $\Omega^{(2)}$ is the same as that with $\Omega^{(1)}$ with the ' $y$ variables renamed.' Hence we only need to consider (17) for only one subset $\Omega$ of $\Omega_{j}$ with the given size. So, for each positive integer $k$ with $k \leq\left|\Omega_{j}\right|$, we only consider the subset $\Omega$ of $\Omega_{j}$ consisting of the first $k$ (smaller) elements of $\Omega_{j}$. We denote this subset by $\Omega_{j k}$, and the empty subset of $\Omega_{j}$ by $\Omega_{j 0}$. From now on, we assume that $\Omega=\Omega_{j k}$ for some integer $k\left(0 \leq k \leq\left|\Omega_{j}\right|=\left(\begin{array}{l}n \\ j\end{array}\right)\right)$. For example, when $n=3$, we see that

$$
\Omega_{20}=\phi, \quad \Omega_{21}=\{\{2,3\}\}, \quad \Omega_{22}=\{\{2,3\},\{1,3\}\}, \quad \Omega_{23}=\{\{2,3\},\{1,3\},\{1,2\}\} .
$$

Further, we assume that we have a decomposition of $\Omega^{(e)}$ as the disjoint union

$$
\Omega^{(e)}=\bigcup_{A \in \Omega_{j}-\Omega} \Omega_{A}^{(e)}
$$

satisfying the following conditions:

(i) $\left|\Omega_{A}^{(e)}\right| \leq\left|\Omega_{A^{\prime}}^{(e)}\right|$ for all $A, A^{\prime} \in \Omega_{j}-\Omega$ with $A<A^{\prime}$,

(ii) $\Omega_{A}^{(e)}<\Omega_{A^{\prime}}^{(e)}$ for all $A, A^{\prime} \in \Omega_{j}-\Omega$ with $A<A^{\prime}$.

(Note: in view of (19), this requires in particular that we should choose $\Omega^{(e)}=\phi$ for $\Omega_{j}-$ $\Omega=\phi$.)

We assume that we are given such a decomposition as in (19) satisfying (i) and (ii). Then we write (17) as

$$
\begin{aligned}
I_{j}(\Omega)= & \frac{1}{\prod_{A \in \Omega} w_{\bar{A}}} \prod_{A \in \Omega_{j}-\Omega} \prod_{E \in \Omega_{A}^{(e)}} \sum_{i_{E}=0}^{w_{\bar{E}}-1} \int_{\mathbb{Z}_{p}} e^{w_{A}\left(x_{\bar{A}}+w_{\bar{A}} y_{\bar{A}}+\sum_{E \in \Omega_{A}^{(e)}} i_{E} \frac{w_{E}}{w_{A}}\right) t} d \mu\left(x_{\bar{A}}\right) \\
& \times \prod_{A \in \Omega^{(s)}} \sum_{j_{A}=0}^{\infty} S_{j_{A}}\left(w_{\bar{A}}-1\right) \frac{\left(w_{A} t\right)^{j_{A}}}{j_{A} !} \\
= & \frac{1}{\prod_{A \in \Omega} w_{\bar{A}}} \prod_{A \in \Omega_{j}-\Omega} \sum_{l_{A}=0}^{\infty} \prod_{E \in \Omega_{A}^{(e)}} \sum_{i_{E}=0}^{w_{\bar{E}}^{-1}} B_{l_{A}}\left(w_{\bar{A}} y_{\bar{A}}+\sum_{E \in \Omega_{A}^{(e)}} i_{E} \frac{w_{E}}{w_{A}}\right) \frac{\left(w_{A} t\right)^{l_{A}}}{l_{A} !} \\
& \times \prod_{A \in \Omega^{(s)}} \sum_{j_{A}=0}^{\infty} S_{j_{A}}\left(w_{\bar{A}}-1\right) \frac{\left(w_{A} t\right)^{j_{A}}}{j_{A} !} .
\end{aligned}
$$

Further, by rearranging sums (20) can be written as

$$
\begin{aligned}
I_{j}(\Omega)= & \frac{1}{\prod_{A \in \Omega} w_{\bar{A}}}\left\{\sum_{n=0}^{\infty} \sum_{\sum_{A \in \Omega_{j}-\Omega} l_{A}+\sum_{A \in \Omega^{(s)}} j_{A}=n}\left(\begin{array}{c}
n \\
\ldots, l_{A}, \ldots, j_{A}, \ldots
\end{array}\right)\right. \\
& \times \prod_{A \in \Omega_{j}-\Omega} \prod_{E \in \Omega_{A}^{(e)}} \sum_{i_{E}=0}^{w_{\bar{E}}-1} B_{l_{A}}\left(w_{\bar{A}} y_{\bar{A}}+\sum_{E \in \Omega_{A}^{(e)}} i_{E} \frac{w_{E}}{w_{A}}\right) \\
& \left.\times \prod_{A \in \Omega^{(s)}} S_{j_{A}}\left(w_{\bar{A}}-1\right) \prod_{A \in \Omega_{j}-\Omega} w_{A}^{l_{A}} \prod_{A \in \Omega^{(s)}} w_{A}^{j_{A}} \frac{t^{n}}{n !}\right\} .
\end{aligned}
$$

Here, $\left(\begin{array}{c}n \\ n \\ l_{A}, \ldots, j_{A}, \ldots\end{array}\right)$ denotes the multinomial coefficient, where $l_{A}$ and $j_{A}$ are nonnegative integers varying respectively over the index sets $A \in \Omega_{j}-\Omega$ and $A \in \Omega^{(s)}$. 
For the next theorem, we assume that $\Omega=\Omega_{j k}$ for some $k, \Omega$ has a decomposition into a disjoint union $\Omega=\Omega^{(e)} \cup \Omega^{(s)}$ with $\Omega^{(e)}<\Omega^{(s)}$, and that $\Omega^{(e)}$ has a decomposition $\Omega^{(e)}=\bigcup_{A \in \Omega_{j}-\Omega} \Omega_{A}^{(e)}$ satisfying conditions (i) and (ii) in (19). As we noted earlier, the $p$-adic integrals in (14) are invariant under every permutation of $w_{1}, w_{2}, \ldots, w_{n}$, so that it gives the identities of symmetry with respect to $w_{1}, w_{2}, \ldots, w_{n}$, involving Bernoulli polynomials and power sums. Now, we have our main result from (21).

Theorem 2.2 The following expression is invariant under every permutation of $w_{1}, w_{2}, \ldots$, $w_{n}$, so that it gives the identities of symmetry with respect to $w_{1}, w_{2}, \ldots, w_{n}$ :

$$
\begin{gathered}
\frac{1}{\prod_{A \in \Omega} w_{\bar{A}}} \sum_{\sum_{A \in \Omega_{j}-\Omega} l_{A}+\sum_{A \in \Omega^{(s)}} j_{A}=n}\left(\begin{array}{c}
n \\
\ldots, l_{A}, \ldots, j_{A}, \ldots
\end{array}\right) \\
\times \prod_{A \in \Omega_{j}-\Omega} \prod_{E \in \Omega_{A}^{(e)}} \sum_{i_{E}=0}^{w_{\bar{E}^{-}}-1} B_{l_{A}}\left(w_{\bar{A}} y_{\bar{A}}+\sum_{E \in \Omega_{A}^{(e)}} i_{E} \frac{w_{E}}{w_{A}}\right) \\
\times \prod_{A \in \Omega^{(s)}} S_{j_{A}}\left(w_{\bar{A}}-1\right) \prod_{A \in \Omega_{j}-\Omega} w_{A}^{l_{A}} \prod_{A \in \Omega^{(s)}} w_{A}^{j_{A}} .
\end{gathered}
$$

In other words, for all permutations $\sigma \in S_{n}$, the following expressions are all the same:

$$
\begin{gathered}
\frac{1}{\prod_{A \in \Omega} w_{\overline{\sigma A}}} \sum_{\sum_{A \in \Omega_{j}-\Omega} l_{A}+\sum_{A \in \Omega^{(s)}} j_{A}=n}\left(\begin{array}{c}
n \\
\ldots, l_{A}, \ldots, j_{A}, \ldots
\end{array}\right) \\
\times \prod_{A \in \Omega_{j}-\Omega_{E \in \Omega_{A}^{(e)}}} \sum_{i_{E}=0}^{w_{\overline{\sigma E}}^{-1}} B_{l_{A}}\left(w_{\overline{\sigma A}} y_{\bar{A}}+\sum_{E \in \Omega_{A}^{(e)}} i_{E} \frac{w_{\sigma E}}{w_{\sigma A}}\right) \\
\times \prod_{A \in \Omega^{(s)}} S_{j_{A}}\left(w_{\overline{\sigma A}}-1\right) \prod_{A \in \Omega_{j}-\Omega} w_{\sigma A}^{l_{A}} \prod_{A \in \Omega^{(s)}} w_{\sigma A}^{j_{A}} .
\end{gathered}
$$

Here, $\left(\begin{array}{c}n \\ n \\ \ldots, l_{A}, \ldots, j_{A}, \ldots\end{array}\right)$ denotes the multinomial coefficient, where $l_{A}$ and $j_{A}$ are nonnegative integers varying respectively over the index sets $A \in \Omega_{j}-\Omega$ and $A \in \Omega^{(s)}$.

\section{Examples}

Here, we would like to illustrate our Theorem 2.2.

Example 3.1 Assume that $n=3, j=2$. Here, $\Omega_{2}=\Omega_{2}^{(3)}=\{\overline{1}=\{2,3\}<\overline{2}=\{1,3\}<\overline{3}=\{1,2\}\}$. In view of our discussion leading up to Theorem 2.2, we may consider only the subsets $\Omega=\Omega_{2 i}(i=0,1,2,3)$ of $\Omega_{2}$.

(a) $\Omega_{20}=\phi\left(\Omega_{2}-\Omega_{20}=\Omega_{2}\right)$

(a-1) $\Omega_{20}^{(e)}=\phi\left(\Omega_{20, A}^{(e)}=\phi\right.$ for each $\left.A \in \Omega_{2}\right), \Omega_{20}^{(s)}=\phi$.

(b) $\Omega_{21}=\{\overline{1}\}\left(\Omega_{2}-\Omega_{21}=\{\overline{2}, \overline{3}\}\right)$

(b-1) $\Omega_{21}^{(e)}=\phi\left(\Omega_{21, A}^{(e)}=\phi\right.$ for each $\left.A \in \Omega_{2}-\Omega_{21}\right), \Omega_{21}^{(s)}=\{\overline{1}\}$,

(b-2) $\Omega_{21}^{(e)}=\{\overline{1}\}\left(\Omega_{21, \overline{2}}^{(e)}=\phi, \Omega_{21, \overline{3}}^{(e)}=\{\overline{1}\}\right), \Omega_{21}^{(s)}=\phi$.

(c) $\Omega_{22}=\{\overline{1}, \overline{2}\}\left(\Omega_{2}-\Omega_{22}=\{\overline{3}\}\right)$

(c-1) $\Omega_{22}^{(e)}=\phi\left(\Omega_{22, \overline{3}}^{(e)}=\phi\right), \Omega_{22}^{(s)}=\{\overline{1}, \overline{2}\}$,

(c-2) $\Omega_{22}^{(e)}=\{\overline{1}\}\left(\Omega_{22, \overline{3}}^{(e)}=\{\overline{1}\}\right), \Omega_{22}^{(s)}=\{\overline{2}\}$,

(c-3) $\Omega_{22}^{(e)}=\{\overline{1}, \overline{2}\}\left(\Omega_{22, \overline{3}}^{(e)}=\{\overline{1}, \overline{2}\}\right), \Omega_{22}^{(s)}=\phi$. 
(d) $\Omega_{23}=\{\overline{1}, \overline{2}, \overline{3}\}\left(\Omega_{2}-\Omega_{23}=\phi\right)$

$(\mathrm{d}-1) \Omega_{23}^{(e)}=\phi, \Omega_{23}^{(s)}=\Omega_{23}$.

One checks now that the invariance of (14) under any permutation of $w_{1}, w_{2}, w_{3}$, applied to each of the cases (a-1), (b-1), (b-2), (c-1), (c-2), and (c-3), yields the results in Theorems $1,2,5,8,11,14$ in [10]. As we noted in [10], not all of these give the full six identities of symmetry corresponding to the symmetric group $S_{3}$. The possible numbers of distinct identities of symmetry are $1,2,3$, and 6 corresponding to the quotient $\left|S_{3}\right| /|H|$, where $H$ is a subgroup of $S_{3}$, with the respective orders $6,3,2$, and 1 . In our case, (a-1), (b-1), (b-2), and (c-2) give the full six identities of symmetry, and (c-1) and (c-3) yield three identities of symmetry, while (d-1) gives no identities of symmetry. For convenience of the reader, we reproduce those results here with appropriate change of notations in (22).

$$
\begin{aligned}
& \sum_{k+\ell+m=n}\left(\begin{array}{c}
n \\
k, \ell, m
\end{array}\right) B_{k}\left(w_{1} y_{1}\right) B_{\ell}\left(w_{2} y_{2}\right) B_{m}\left(w_{3} y_{3}\right) w_{1}^{\ell+m} w_{2}^{k+m} w_{3}^{k+\ell} \\
& =\sum_{k+\ell+m=n}\left(\begin{array}{c}
n \\
k, \ell, m
\end{array}\right) B_{k}\left(w_{1} y_{1}\right) B_{\ell}\left(w_{3} y_{2}\right) B_{m}\left(w_{2} y_{3}\right) w_{1}^{\ell+m} w_{3}^{k+m} w_{2}^{k+\ell} \\
& =\sum_{k+\ell+m=n}\left(\begin{array}{c}
n \\
k, \ell, m
\end{array}\right) B_{k}\left(w_{2} y_{1}\right) B_{\ell}\left(w_{1} y_{2}\right) B_{m}\left(w_{3} y_{3}\right) w_{2}^{\ell+m} w_{1}^{k+m} w_{3}^{k+\ell} \\
& =\sum_{k+\ell+m=n}\left(\begin{array}{c}
n \\
k, \ell, m
\end{array}\right) B_{k}\left(w_{2} y_{1}\right) B_{\ell}\left(w_{3} y_{2}\right) B_{m}\left(w_{1} y_{3}\right) w_{2}^{\ell+m} w_{3}^{k+m} w_{1}^{k+\ell} \\
& =\sum_{k+\ell+m=n}\left(\begin{array}{c}
n \\
k, \ell, m
\end{array}\right) B_{k}\left(w_{3} y_{1}\right) B_{\ell}\left(w_{1} y_{2}\right) B_{m}\left(w_{2} y_{3}\right) w_{3}^{\ell+m} w_{1}^{k+m} w_{2}^{k+\ell} \\
& =\sum_{k+\ell+m=n}\left(\begin{array}{c}
n \\
k, \ell, m
\end{array}\right) B_{k}\left(w_{3} y_{1}\right) B_{\ell}\left(w_{2} y_{2}\right) B_{m}\left(w_{1} y_{3}\right) w_{3}^{\ell+m} w_{2}^{k+m} w_{1}^{k+\ell}, \\
& \sum_{k+\ell+m=n}\left(\begin{array}{c}
n \\
k, \ell, m
\end{array}\right) B_{k}\left(w_{2} y_{2}\right) B_{\ell}\left(w_{3} y_{3}\right) S_{m}\left(w_{1}-1\right) w_{2}^{\ell+m} w_{3}^{k+m} w_{1}^{k+\ell-1} \\
& =\sum_{k+\ell+m=n}\left(\begin{array}{c}
n \\
k, \ell, m
\end{array}\right) B_{k}\left(w_{2} y_{2}\right) B_{\ell}\left(w_{1} y_{3}\right) S_{m}\left(w_{3}-1\right) w_{2}^{\ell+m} w_{1}^{k+m} w_{3}^{k+\ell-1} \\
& =\sum_{k+\ell+m=n}\left(\begin{array}{c}
n \\
k, \ell, m
\end{array}\right) B_{k}\left(w_{1} y_{2}\right) B_{\ell}\left(w_{3} y_{3}\right) S_{m}\left(w_{2}-1\right) w_{1}^{\ell+m} w_{3}^{k+m} w_{2}^{k+\ell-1} \\
& =\sum_{k+\ell+m=n}\left(\begin{array}{c}
n \\
k, \ell, m
\end{array}\right) B_{k}\left(w_{1} y_{2}\right) B_{\ell}\left(w_{2} y_{3}\right) S_{m}\left(w_{3}-1\right) w_{1}^{\ell+m} w_{2}^{k+m} w_{3}^{k+\ell-1} \\
& =\sum_{k+\ell+m=n}\left(\begin{array}{c}
n \\
k, \ell, m
\end{array}\right) B_{k}\left(w_{3} y_{2}\right) B_{\ell}\left(w_{2} y_{3}\right) S_{m}\left(w_{1}-1\right) w_{3}^{\ell+m} w_{2}^{k+m} w_{1}^{k+\ell-1} \\
& =\sum_{k+\ell+m=n}\left(\begin{array}{c}
n \\
k, \ell, m
\end{array}\right) B_{k}\left(w_{3} y_{2}\right) B_{\ell}\left(w_{1} y_{3}\right) S_{m}\left(w_{2}-1\right) w_{3}^{\ell+m} w_{1}^{k+m} w_{2}^{k+\ell-1}, \\
& w_{1}^{n-1} \sum_{k=0}^{n}\left(\begin{array}{l}
n \\
k
\end{array}\right) B_{k}\left(w_{2} y_{2}\right) \sum_{i=0}^{w_{1}-1} B_{n-k}\left(w_{3} y_{3}+\frac{w_{3}}{w_{1}} i\right) w_{2}^{n-k} w_{3}^{k} \\
& =w_{1}^{n-1} \sum_{k=0}^{n}\left(\begin{array}{l}
n \\
k
\end{array}\right) B_{k}\left(w_{3} y_{2}\right) \sum_{i=0}^{w_{1}-1} B_{n-k}\left(w_{2} y_{3}+\frac{w_{2}}{w_{1}} i\right) w_{3}^{n-k} w_{2}^{k}
\end{aligned}
$$




$$
\begin{aligned}
& =w_{2}^{n-1} \sum_{k=0}^{n}\left(\begin{array}{l}
n \\
k
\end{array}\right) B_{k}\left(w_{1} y_{2}\right) \sum_{i=0}^{w_{2}-1} B_{n-k}\left(w_{3} y_{3}+\frac{w_{3}}{w_{2}} i\right) w_{1}^{n-k} w_{3}^{k} \\
& =w_{2}^{n-1} \sum_{k=0}^{n}\left(\begin{array}{l}
n \\
k
\end{array}\right) B_{k}\left(w_{3} y_{2}\right) \sum_{i=0}^{w_{2}-1} B_{n-k}\left(w_{1} y_{3}+\frac{w_{1}}{w_{2}} i\right) w_{3}^{n-k} w_{1}^{k} \\
& =w_{3}^{n-1} \sum_{k=0}^{n}\left(\begin{array}{l}
n \\
k
\end{array}\right) B_{k}\left(w_{1} y_{2}\right) \sum_{i=0}^{w_{3}-1} B_{n-k}\left(w_{2} y_{3}+\frac{w_{2}}{w_{3}} i\right) w_{1}^{n-k} w_{2}^{k} \\
& =w_{3}^{n-1} \sum_{k=0}^{n}\left(\begin{array}{l}
n \\
k
\end{array}\right) B_{k}\left(w_{2} y_{2}\right) \sum_{i=0}^{w_{3}-1} B_{n-k}\left(w_{1} y_{3}+\frac{w_{1}}{w_{3}} i\right) w_{2}^{n-k} w_{1}^{k} \\
& \sum_{k+\ell+m=n}\left(\begin{array}{c}
n \\
k, \ell, m
\end{array}\right) B_{k}\left(w_{3} y_{3}\right) S_{\ell}\left(w_{1}-1\right) S_{m}\left(w_{2}-1\right) w_{3}^{\ell+m} w_{1}^{k+m-1} w_{2}^{k+\ell-1} \\
& =\sum_{k+\ell+m=n}\left(\begin{array}{c}
n \\
k, \ell, m
\end{array}\right) B_{k}\left(w_{1} y_{3}\right) S_{\ell}\left(w_{2}-1\right) S_{m}\left(w_{3}-1\right) w_{1}^{\ell+m} w_{2}^{k+m-1} w_{3}^{k+\ell-1} \\
& =\sum_{k+\ell+m=n}\left(\begin{array}{c}
n \\
k, \ell, m
\end{array}\right) B_{k}\left(w_{2} y_{3}\right) S_{\ell}\left(w_{1}-1\right) S_{m}\left(w_{3}-1\right) w_{2}^{\ell+m} w_{1}^{k+m-1} w_{3}^{k+\ell-1}, \\
& w_{1}^{n-1} \sum_{k=0}^{n}\left(\begin{array}{l}
n \\
k
\end{array}\right) \sum_{i=0}^{w_{1}-1} B_{k}\left(w_{3} y_{3}+\frac{w_{3}}{w_{1}} i\right) S_{n-k}\left(w_{2}-1\right) w_{3}^{n-k} w_{2}^{k-1} \\
& =w_{2}^{n-1} \sum_{k=0}^{n}\left(\begin{array}{l}
n \\
k
\end{array}\right) \sum_{i=0}^{w_{2}-1} B_{k}\left(w_{3} y_{3}+\frac{w_{3}}{w_{2}} i\right) S_{n-k}\left(w_{1}-1\right) w_{3}^{n-k} w_{1}^{k-1} \\
& =w_{2}^{n-1} \sum_{k=0}^{n}\left(\begin{array}{l}
n \\
k
\end{array}\right) \sum_{i=0}^{w_{2}-1} B_{k}\left(w_{1} y_{3}+\frac{w_{1}}{w_{2}} i\right) S_{n-k}\left(w_{3}-1\right) w_{1}^{n-k} w_{3}^{k-1} \\
& =w_{3}^{n-1} \sum_{k=0}^{n}\left(\begin{array}{l}
n \\
k
\end{array}\right) \sum_{i=0}^{w_{3}-1} B_{k}\left(w_{1} y_{3}+\frac{w_{1}}{w_{3}} i\right) S_{n-k}\left(w_{2}-1\right) w_{1}^{n-k} w_{2}^{k-1} \\
& =w_{1}^{n-1} \sum_{k=0}^{n}\left(\begin{array}{l}
n \\
k
\end{array}\right) \sum_{i=0}^{w_{1}-1} B_{k}\left(w_{2} y_{3}+\frac{w_{2}}{w_{1}} i\right) S_{n-k}\left(w_{3}-1\right) w_{2}^{n-k} w_{3}^{k-1} \\
& =w_{3}^{n-1} \sum_{k=0}^{n}\left(\begin{array}{l}
n \\
k
\end{array}\right) \sum_{i=0}^{w_{3}-1} B_{k}\left(w_{2} y_{3}+\frac{w_{2}}{w_{3}} i\right) S_{n-k}\left(w_{1}-1\right) w_{2}^{n-k} w_{1}^{k-1}, \\
& \left(w_{1} w_{2}\right)^{n-1} \sum_{i=0}^{w_{1}-1} \sum_{j=0}^{w_{2}-1} B_{n}\left(w_{3} y_{3}+\frac{w_{3}}{w_{1}} i+\frac{w_{3}}{w_{2}} j\right) \\
& =\left(w_{2} w_{3}\right)^{n-1} \sum_{i=0}^{w_{2}-1} \sum_{j=0}^{w_{3}-1} B_{n}\left(w_{1} y_{3}+\frac{w_{1}}{w_{2}} i+\frac{w_{1}}{w_{3}} j\right) \\
& =\left(w_{3} w_{1}\right)^{n-1} \sum_{i=0}^{w_{3}-1} \sum_{j=0}^{w_{1}-1} B_{n}\left(w_{2} y_{3}+\frac{w_{2}}{w_{3}} i+\frac{w_{2}}{w_{1}} j\right) .
\end{aligned}
$$

Example 3.2 Assume that $n=4, j=3$. Here, $\Omega_{3}=\Omega_{3}^{(4)}=\{\overline{1}=\{2,3,4\}<\overline{2}=\{1,3,4\}<\overline{3}=$ $\{1,2,4\}<\overline{4}=\{1,2,3\}\}$. In view of our discussion leading up to Theorem 2.2, we may con- 
sider only the subsets $\Omega=\Omega_{3 i}(i=0,1,2,3,4)$ of $\Omega_{3}$. We let the interested reader write out the identities of symmetry for each of the following cases by using Theorem 2.2.

(a) $\Omega_{30}=\phi\left(\Omega_{3}-\Omega_{30}=\Omega_{3}\right)$

(a-1) $\Omega_{30}^{(e)}=\phi\left(\Omega_{30, A}^{(e)}=\phi\right.$ for each $\left.A \in \Omega_{3}\right), \Omega_{30}^{(s)}=\phi$.

(b) $\Omega_{31}=\{\overline{1}\}\left(\Omega_{3}-\Omega_{31}=\{\overline{2}, \overline{3}, \overline{4}\}\right)$

(b-1) $\Omega_{31}^{(e)}=\phi\left(\Omega_{31, A}^{(e)}=\phi\right.$ for each $\left.A \in \Omega_{3}-\Omega_{31}\right), \Omega_{31}^{(s)}=\{\overline{1}\}$,

(b-2) $\Omega_{31}^{(e)}=\{\overline{1}\}\left(\Omega_{31, \overline{2}}^{(e)}=\Omega_{31, \overline{3}}^{(e)}=\phi, \Omega_{31, \overline{4}}^{(e)}=\{\overline{1}\}\right), \Omega_{31}^{(s)}=\phi$.

(c) $\Omega_{32}=\{\overline{1}, \overline{2}\}\left(\Omega_{3}-\Omega_{32}=\{\overline{3}, \overline{4}\}\right)$

(c-1) $\Omega_{32}^{(e)}=\phi\left(\Omega_{32, A}^{(e)}=\phi\right.$ for each $\left.A \in \Omega_{3}-\Omega_{32}\right), \Omega_{32}^{(s)}=\{\overline{1}, \overline{2}\}$,

(c-2) $\Omega_{32}^{(e)}=\{\overline{1}\}\left(\Omega_{32, \overline{3}}^{(e)}=\phi, \Omega_{32, \overline{4}}^{(e)}=\{1\}\right), \Omega_{32}^{(s)}=\{\overline{2}\}$,

$(\mathrm{c}-3-1) \quad \Omega_{32}^{(e)}=\{\overline{1}, \overline{2}\}\left(\Omega_{32, \overline{3}}^{(e)}=\phi, \Omega_{32, \overline{4}}^{(e)}=\{\overline{1}, \overline{2}\}\right), \Omega_{32}^{(s)}=\phi$,

$(\mathrm{c}-3-2) \Omega_{32}^{(e)}=\{\overline{1}, \overline{2}\}\left(\Omega_{32, \overline{3}}^{(e)}=\{\overline{1}\}, \Omega_{32, \overline{4}}^{(e)}=\{\overline{2}\}\right), \Omega_{32}^{(s)}=\phi$.

(d) $\Omega_{34}=\{\overline{1}, \overline{2}, \overline{3}, \overline{4}\}\left(\Omega_{3}-\Omega_{34}=\phi\right)$

$(\mathrm{d}-1) \Omega_{34}^{(e)}=\phi, \Omega_{34}^{(s)}=\Omega_{34}$.

Example 3.3 Assume that $n=4, j=2$. Here, $\Omega_{2}=\Omega_{2}^{(4)}=\{34=\{3,4\}<24=\{2,4\}<23=$ $\{2,3\}<14=\{1,4\}<13=\{1,3\}<12=\{1,2\}\}$. In view of our discussion leading up to Theorem 2.2, we may consider only the subsets $\Omega=\Omega_{2 i}(i=0,1,2,3,4,5,6)$ of $\Omega_{2}$. We let the interested reader write out the identities of symmetry for each of the following cases by using Theorem 2.2.

(a) $\Omega_{20}=\phi\left(\Omega_{2}-\Omega_{20}=\Omega_{2}\right)$

(a-1) $\Omega_{20}^{(e)}=\phi\left(\Omega_{20, A}^{(e)}=\phi\right.$ for each $\left.A \in \Omega_{2}\right), \Omega_{20}^{(s)}=\phi$.

(b) $\Omega_{21}=\{34\}\left(\Omega_{2}-\Omega_{21}=\{24,23,14,13,12\}\right)$

(b-1) $\Omega_{21}^{(e)}=\phi\left(\Omega_{21, A}^{(e)}=\phi\right.$ for each $\left.A \in \Omega_{2}-\Omega_{21}\right), \Omega_{21}^{(s)}=\{34\}$,

(b-2) $\Omega_{21}^{(e)}=\{34\}\left(\Omega_{21, A}^{(e)}=\phi\right.$ for each $\left.A \in\{24,23,14,13\}, \Omega_{21,12}^{(e)}=\{34\}\right), \Omega_{21}^{(s)}=\phi$.

(c) $\Omega_{22}=\{34,24\}\left(\Omega_{2}-\Omega_{22}=\{23,14,13,12\}\right)$

(c-1) $\Omega_{22}^{(e)}=\phi\left(\Omega_{22, A}^{(e)}=\phi\right.$ for each $\left.A \in \Omega_{2}-\Omega_{22}\right), \Omega_{22}^{(s)}=\{34,24\}$,

(c-2) $\Omega_{22}^{(e)}=\{34\}\left(\Omega_{22,23}^{(e)}=\Omega_{22,14}^{(e)}=\Omega_{22,13}^{(e)}=\phi, \Omega_{22,12}^{(e)}=\{34\}\right), \Omega_{22}^{(s)}=\{24\}$,

(c-3-1) $\Omega_{22}^{(e)}=\{34,24\}\left(\Omega_{22,23}^{(e)}=\Omega_{22,14}^{(e)}=\Omega_{22,13}^{(e)}=\phi, \Omega_{22,12}^{(e)}=\{34,24\}\right), \Omega_{22}^{(s)}=\phi$,

$(\mathrm{c}-3-2) \Omega_{22}^{(e)}=\{34,24\}\left(\Omega_{22,23}^{(e)}=\Omega_{22,14}^{(e)}=\phi, \Omega_{22,13}^{(e)}=\{34\}, \Omega_{22,12}^{(e)}=\{24\}\right)$,

$$
\Omega_{22}^{(s)}=\phi \text {. }
$$

(d) $\Omega_{23}=\{34,24,23\}\left(\Omega_{2}-\Omega_{23}=\{14,13,12\}\right)$

(d-1) $\Omega_{23}^{(e)}=\phi\left(\Omega_{23, A}^{(e)}=\phi\right.$ for each $\left.A \in\{14,13,12\}\right), \Omega_{23}^{(s)}=\{34,24,23\}$,

$(\mathrm{d}-2) \Omega_{23}^{(e)}=\{34\}\left(\Omega_{23,14}^{(e)}=\Omega_{23,13}^{(e)}=\phi, \Omega_{23,12}^{(e)}=\{34\}\right), \Omega_{23}^{(s)}=\{24,23\}$,

$(\mathrm{d}-3-1) \Omega_{23}^{(e)}=\{34,24\}\left(\Omega_{23,14}^{(e)}=\Omega_{23,13}^{(e)}=\phi, \Omega_{23,12}^{(e)}=\{34,24\}\right), \Omega_{23}^{(s)}=\{23\}$,

$(\mathrm{d}-3-2) \Omega_{23}^{(e)}=\{34,24\}\left(\Omega_{23,14}^{(e)}=\phi, \Omega_{23,13}^{(e)}=\{34\}, \Omega_{23,12}^{(e)}=\{24\}\right), \Omega_{23}^{(s)}=\{23\}$,

$(\mathrm{d}-4-1) \Omega_{23}^{(e)}=\{34,24,23\}\left(\Omega_{23,14}^{(e)}=\Omega_{23,13}^{(e)}=\phi, \Omega_{23,12}^{(e)}=\{34,24,23\}\right), \Omega_{23}^{(s)}=\phi$,

$(\mathrm{d}-4-2) \Omega_{23}^{(e)}=\{34,24,23\}\left(\Omega_{23,14}^{(e)}=\phi, \Omega_{23,13}^{(e)}=\{34\}, \Omega_{23,12}^{(e)}=\{24,23\}\right), \Omega_{23}^{(s)}=\phi$,

$(\mathrm{d}-4-3) \Omega_{23}^{(e)}=\{34,24,23\}\left(\Omega_{23,14}^{(e)}=\{34\}, \Omega_{23,13}^{(e)}=\{24\}, \Omega_{23,12}^{(e)}=\{23\}\right), \Omega_{23}^{(s)}=\phi$.

(e) $\Omega_{24}=\{34,24,23,14\}\left(\Omega_{2}-\Omega_{24}=\{13,12\}\right)$

(e-1) $\Omega_{24}^{(e)}=\phi\left(\Omega_{24,13}^{(e)}=\Omega_{24,12}^{(e)}=\phi\right), \Omega_{24}^{(s)}=\{34,24,23,14\}$,

(e-2) $\Omega_{24}^{(e)}=\{34\}\left(\Omega_{24,13}^{(e)}=\phi, \Omega_{24,12}^{(e)}=\{34\}\right), \Omega_{24}^{(s)}=\{24,23,14\}$,

(e-3-1) $\Omega_{24}^{(e)}=\{34,24\}\left(\Omega_{24,13}^{(e)}=\phi, \Omega_{24,12}^{(e)}=\{34,24\}\right), \Omega_{24}^{(s)}=\{23,14\}$,

(e-3-2) $\Omega_{24}^{(e)}=\{34,24\}\left(\Omega_{24,13}^{(e)}=\{34\}, \Omega_{24,12}^{(e)}=\{24\}\right), \Omega_{24}^{(s)}=\{23,14\}$,

(e-4-1) $\Omega_{24}^{(e)}=\{34,24,23\}\left(\Omega_{24,13}^{(e)}=\phi, \Omega_{24,12}^{(e)}=\{34,24,23\}\right), \Omega_{24}^{(s)}=\{14\}$,

$(\mathrm{e}-4-2) \Omega_{24}^{(e)}=\{34,24,23\}\left(\Omega_{24,13}^{(e)}=\{34\}, \Omega_{24,12}^{(e)}=\{24,23\}\right), \Omega_{24}^{(s)}=\{14\}$, 


$$
\begin{aligned}
& (\mathrm{e}-5-1) \Omega_{24}^{(e)}=\{34,24,23,14\}\left(\Omega_{24,13}^{(e)}=\phi, \Omega_{24,12}^{(e)}=\{34,24,23,14\}\right), \Omega_{24}^{(s)}=\phi, \\
& (\mathrm{e}-5-2) \Omega_{24}^{(e)}=\{34,24,23,14\}\left(\Omega_{24,13}^{(e)}=\{34\}, \Omega_{24,12}^{(e)}=\{24,23,14\}\right), \Omega_{24}^{(s)}=\phi, \\
& (\mathrm{e}-5-3) \Omega_{24}^{(e)}=\{34,24,23,14\}\left(\Omega_{24,13}^{(e)}=\{34,24\}, \Omega_{24,12}^{(e)}=\{23,14\}\right), \Omega_{24}^{(s)}=\phi .
\end{aligned}
$$

(f) $\Omega_{25}=\{34,24,23,14,13\}\left(\Omega_{2}-\Omega_{25}=\{12\}\right)$

$(\mathrm{f}-1) \Omega_{25}^{(e)}=\phi\left(\Omega_{25,12}^{(e)}=\phi\right), \Omega_{25}^{(s)}=\{34,24,23,14,13\}$,

$(\mathrm{f}-2) \Omega_{25}^{(e)}=\{34\}\left(\Omega_{25,12}^{(e)}=\{34\}\right), \Omega_{25}^{(s)}=\{24,23,14,13\}$,

(f-3) $\Omega_{25}^{(e)}=\{34,24\}\left(\Omega_{25,12}^{(e)}=\{34,24\}\right), \Omega_{25}^{(s)}=\{23,14,13\}$,

(f-4) $\Omega_{25}^{(e)}=\{34,24,23\}\left(\Omega_{25,12}^{(e)}=\{34,24,23\}\right), \Omega_{25}^{(s)}=\{14,13\}$,

$(\mathrm{f}-5) \Omega_{25}^{(e)}=\{34,24,23,14\}\left(\Omega_{25,12}^{(e)}=\{34,24,23,14\}\right), \Omega_{25}^{(s)}=\{13\}$,

$(\mathrm{f}-6) \Omega_{25}^{(e)}=\{34,24,23,14,13\}\left(\Omega_{25,12}^{(e)}=\{34,24,23,14,13\}\right), \Omega_{25}^{(s)}=\phi$.

(g) $\Omega_{26}=\{34,24,23,14,13,12\}\left(\Omega_{2}-\Omega_{26}=\phi\right)$

$(\mathrm{g}-1) \Omega_{26}^{(e)}=\phi, \Omega_{26}^{(s)}=\Omega_{26}$.

\section{Conclusion}

Identities of symmetry in two variables for Bernoulli polynomials and power sums, which had been shown by using suitable symmetric identities, were derived by employing a completely different tool in [12], namely the $p$-adic Volkenborn integrals. Not much later, it was observed in [10] that the identities in two variables can be extended to those in three variables. We recalled that the abundant symmetries of identities in three variables shed new light even on the existing identities in two variables. Namely, some further identities of symmetry can be discovered by specializing one of the three variables as 1 .

Here, in this paper, we generalized the results in three variables to those in an arbitrary number of variables in a suitable setting. We proved our main result, Theorem 2.2, and illustrated our result with some examples. As was noted in [10] and recalled in Example 3.1 , the number of distinct identities of symmetry in three variables is not always $6=\left|S_{3}\right|$, but it is $1,2,3$, or 6, because it is equal to the quotient $\left|S_{3}\right| /|H|$, where $H$ is a subgroup of $S_{3}$. It is an interesting problem to determine the possible numbers of distinct symmetries in our case. We leave this as a challenging problem for the interested reader. Similar results for other special polynomials together with the corresponding suitable power sums will appear in forthcoming papers.

Acknowledgements

The authors would like to thank Jangjeon Institute for Mathematical Science for the support of this research.

Funding

This work was supported by the National Research Foundation of Korea (NRF)grant funded by the Korea government (No. 2020R1F1A1A01071564).

Availability of data and materials

Not applicable.

Competing interests

The authors declare that they have no competing interests.

Authors' contributions

TK and DSK conceived of the framework and structured the whole paper; DSK and TK wrote the paper; JK and HYK checked the results of the paper and typed the paper; DSK and TK completed the revision of the article. All authors have read and agreed to the published version of the manuscript.

\section{Author details}

'Department of Mathematics, Kwangwoon University, Seoul 139-701, Republic of Korea. ²Department of Mathematics, Sogang University, Seoul 121-742, Republic of Korea. ${ }^{3}$ Department of Mathematics Education and ERI, Gyeongsang National University, Jinju 52828, Republic of Korea. 


\section{Publisher's Note}

Springer Nature remains neutral with regard to jurisdictional claims in published maps and institutional affiliations.

Received: 10 October 2020 Accepted: 9 November 2020 Published online: 18 November 2020

\section{References}

1. Araci, S., Duran, U., Acikgoz, M.: A study on a class of $q$-Euler polynomials under the symmetric group of degree $n$. J. Nonlinear Sci. Appl. 9(8), 5196-5201 (2016)

2. Deeba, E., Rodriguez, D.: Stirling's and Bernoulli numbers. Am. Math. Mon. 98, 423-426 (1991)

3. Gessel, l.: Solution to problem E3237. Am. Math. Mon. 96, 364 (1989)

4. He, Y: Symmetric identities for Carlitz's q-Bernoulli numbers and polynomials. Adv. Differ. Equ. 2013, 246 (2013)

5. Jang, L.-C., Kim, D. S., Kim, T., Lee, H.: p-Adic integral on $\mathbb{Z}_{p}$ associated with degenerate Bernoulli polynomials of the second kind. Adv. Differ. Equ. 2020, 278 (2020)

6. Kim, D.S., Kim, T.: Three variable symmetric identities involving Carlitz-type $q$-Euler polynomials. Math. Sci. 8(4), 147-152 (2014)

7. Kim, D.S., Lee, N., Na, J., Park, K.H.: Abundant symmetry for higher-order Bernoulli polynomials (I). Adv. Stud. Contemp. Math. (Kyungshang) 23(3), 461-482 (2013)

8. Kim, D.S., Lee, N., Na, J., Park, K.H.: Abundant symmetry for higher-order Bernoulli polynomials (II). Proc. Jangjeon Math. Soc. 16(3), 359-378 (2013)

9. Kim, D.S., Park, K.H.: Identities of symmetry for Euler polynomials arising from quotients of fermionic integrals invariant under S. J. Inequal. Appl. 2010, Article ID 851521 (2010)

10. Kim, D.S., Park, K.H.: Identities of symmetry for Bernoulli polynomials arising from quotients of Volkenborn integrals invariant under $S_{3}$. Appl. Math. Comput. 219(10), 5096-5104 (2013)

11. Kim, T: $q$-Volkenborn integration. Russ. J. Math. Phys. 9(3), 288-299 (2002)

12. Kim, T.: Symmetry $p$-adic invariant integral on $\mathbb{Z}_{p}$ for Bernoulli and Euler polynomials. J. Adv. Differ. Equ. Appl. 14, 1267-1277 (2008)

13. Kim, T., Dolgy, D.V., Kim, D.S.: Some identities of $q$-Bernoulli polynomials under symmetry group $S_{3}$. J. Nonlinear Convex Anal. 16(9), 1869-1880 (2015)

14. Kim, T., Kim, D. S., Dolgy, D.V., Pyo, S.-S.: Identities of symmetry for type 2 -Bernoulli polynomials under symmetric group S. J. Nonlinear Convex Anal. 21(9), 1973-1979 (2020)

15. Schikhof, W.H.: Ultrametric Calculus: An Introduction to $p$-Adic Analysis. Cambridge University Press, Cambridge (2006)

16. Simsek, Y.: Complete sum of products of $(h, q)$-extension of Euler polynomials and numbers. J. Differ. Equ. Appl. 16(11), 1331-1348 (2010)

17. Tuenter, H.: A symmetry of power sum polynomials and Bernoulli numbers. Am. Math. Mon. 108, 258-261 (2001)

18. Yang, S.: An identity of symmetry for the Bernoulli polynomials. Discrete Math. 308, 550-554 (2008)

\section{Submit your manuscript to a SpringerOpen ${ }^{\circ}$ journal and benefit from:}

- Convenient online submission

- Rigorous peer review

- Open access: articles freely available online

- High visibility within the field

- Retaining the copyright to your article 